other three dimensions. We believe that a gap of one day between both measurements is justifiable to obtain test-retest reliability of the CRQ in this particular group of patients.

Perhaps the most fundamental misunderstanding is that our research was focused on the prediction of a specific criterion (criterion validity) based on the scores of the CRQ. In our research we investigated the validity of the four dimensions of the CRQ by relating these dimensions to comparable dimensions of the SCL-90. Thus, the principal focus was not on the prediction of a criterion (according to Mrs Jones, the scores of the SCL-90), but on the ability of the CRQ to measure the four dimensions. From a methodological point of view it should be noted that the scores on the CRQ are based on psychological properties of individuals. As a consequence it is often impossible to find a single satisfactory real world criterion available for the evaluation of its validity. The SCL-90 can never serve as such a naturally occurring criterion. Content validity and construct validity are powerful tools to establish our understanding of what dimensions of specific measurement instruments intend to measure, and that was, indeed, the core issue of our research.

PJ WIJKSTRA Asthma Centre, Beatrixoord Hospital, 9751ND Haren, The Netherlands

1 Nunnally J. Psychometric theory. 2nd edn. New York: McGraw-Hill, 1978:7.

2 Allen MJ, Yen WM. Introduction to measurement theory. Belmont. California: Brooks/Cole, 1979.

\section{Impact of HIV on tuberculosis in developing countries}

The advent of HIV has considerably affected tuberculosis control programmes in developing countries as outlined by Drs Nunn, Eliott, and McAdam (May 1994; 49:511-8). In Blantyre, Malawi, for example, the number of notified new cases of tuberculosis has increased fourfold between 1986 and 1993. The brunt of this increase has been borne by young adults and children.

Nunn and colleagues comment on the disproportionate increase in smear negative pulmonary tuberculosis in Malawi. Although in $198690 \%$ of new cases of pulmonary tuberculosis in adults aged between 15 and 44 in Blantyre were AAFB smear positive, this fell to $33 \%$ in 1991 . Possible reasons for this change included the association of HIV with an increase in smear negative pulmonary tuberculosis, a breakdown in the sputum microscopy service or, alternatively, the occurrence of other HIV associated pulmonary pathogens mimicking tuberculosis. ${ }^{1}$

We investigated 82 patients with sputum smear negative, but clinically suspected, pulmonary tuberculosis by the method of sputum induction. ${ }^{2}$ The induced sputum was examined for Mycobacterium tuberculosis and other significant pathogens. Tuberculosis was confirmed by culture in 30 . The only other significant pathogens detected were Pneumocystis carinii in one patient and Strongyloides stercoralis in a second. Both patients were HIV seropositive. We concluded that, although other HIV associated pathogens do occur in
Malawi, they appear to be currently uncommon.

During 1992 intensive efforts were made to ensure that all patients had a proper sputum examination for AAFB. By the end of 1992 the proportion of young adults with smear positive pulmonary tuberculosis had risen from 33 to 60 . This re-emphasises the vital importance of a good sputum microscopy service in a tuberculosis control programme, even in areas with a high incidence of HIV infection.

This work was supported in part by the ScaddingMorriston Davies Fellowship.

C M PARRY

Centre for Tropical Microbiology, Liverpool University, Liverpool L69 3BX, UK O KAMOTO A D HARRIES Department of Medicine, Queen Elizabeth Central Hospital, $P O$ Box 95,
Blantyre,

L NYONG'ONYA MBEWE DS NYANGULU

Malawi National Tuberculosis Control Programme,

Llongwe, Malawi

1 Daley CL. Pulmonary infections in the tropics: impact of HIV infection. Thorax 1994;49:3708.

2 Parry CM, Kamoto O, Harries AD, Wirima JJ, Nyirenda CM, Nyangulu DS, et al. Use of sputum induction for establishing a diagnosis in patients with suspected pulmonary tuberculosis patients with suspected pulmonary
in Malawi. Thorax 1993;48:1079.

\section{Ultrasound assessment of diaphragmatic movement}

I read the paper by Houston and colleagues (May 1994:49:500-3) with interest. A similar technique was used to answer the question "how does the diaphragm move in association with the induction of anaesthesia?". This question was stimulated by a study of three volunteers using lateral radiographs. ${ }^{1}$ The reproducibility of the ultrasound measurements that were made was assessed by asking the operator to identify the precise time when the diaphragm passed through a particular position. ${ }^{2}$ Volunteers were asked to breathe while holding their rib cage dimensions constant, so that the diaphragm position could be measured exactly from a spirometer. The ultrasound operator was able to judge diaphragmatic position with a standard deviation of 15,39 , and $56 \mathrm{ml}$ in three volunteer subjects. I believe that these values, although obtained in a single operator, compare well with the reproducibility values in the more recent study.

In contrast to previous radiological observations in three subjects, the study using ultrasound indicated that motion of the diaphragm dome in a cranial direction, associated with the induction of anaesthesia, was very small. In eight out of 20 subjects no cranial motion was detected. Because the dimensions of the rib cage may also change at the induction of anaesthesia, exact inferences regarding the contribution of diaphragmatic movement to changes in lung volume in these circumstances cannot be made with certainty. It is possible that ultrasound, by allowing measurement of a specific part of the diaphragm, is a better method than radiography, where the image consists of several overlapping shadows.

In the study of Houston and colleagues the reduction in diaphragmatic movement per change in lung volume, observed when the subject moved from the supine to sitting position, presumably indicates the greater action of the diaphragm on the rib cage in that position.

GORDON DRUMMOND Department of Anaesthetics, Edinburgh EH3 9YW, UK

1 Froese AB, Bryan AC. Effects of anesthesia and paralysis in diaphragmatic movements in man. Anesthesiology, 1974;41:242-53.

2 Drummond GB, Allan PL, Logan MR. Changes in diaphragmatic position in association with the induction of anaesthesia. Brf Anaesth 1986, 58:1246-51.

\section{Bordetella bronchiseptica pneumonia}

De la Fuente et al (July 1994;49:719-20) report a case of pneumonia caused by Bordetella bronchiseptica in a patient with AIDS. Human disease, although rare, was reported as early as 1911 in a laboratory worker at the Royal College of Physicians, Edinburgh with an upper respiratory tract infection. ${ }^{1}$ However, infection in association with AIDS may become a subject of increasing importance. The authors suggest exposure to ill animals should raise clinical suspicion. Although clinical disease has been reported in various animals from cats to skunks and racoons, ${ }^{2}$ it should be noted that outwardly asymptomatic carriage occurs.

Unusual lung permeability measurements and evidence of minor foci of haemorrhage and inflammation in the lungs of rabbits supplied from a large commercial source led me to search for infection. All such animals grew $B$ bronchiseptica from bronchoalveolar lavage fluid, though none showed clinical disease before death or evidence of other infection. Discussion with the suppliers revealed that screening of their stocks showed a $45 \%$ carriage rate although all appeared clinically healthy. Other laboratories using the animals had not experienced difficulties with the animals' overall health, suggesting that changes were local and subtle. The suppliers believe that carriage is easily transmitted and that its eradication would require complete restocking of their rabbits so they had no plans to attempt its elimination.

In patients with AIDS and other causes of immunosuppression with pneumonia a history of close contact with any mammal without regard to its health should be sufficient to raise suspicion.

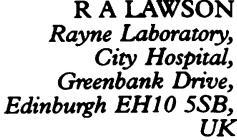

1 McGowan JP. Some observations on a laboratory epidemic principally among dogs and cats, in
which the animals affected presented symptoms of the disease called 'distemper'. $¥$ Pathol Bacteriol 1911;15:372-426.

2 Goodnow RA. Biology of Bordetella bronchiseptica. Microbiol Rev 1980;44:722-38. 